BRIEF COMMUNICATION

\title{
Codonopsis pilosula twines either to the left or to the right
}

${ }^{1}$ Key Laboratory of Adaptation and Evolution of Plateau Biota, Northwest Institute of

5 Plateau Biology, Chinese Academy of Sciences, Xining 810008, China. ${ }^{2}$ Qinghai

6 University, Xining 810016, China. ${ }^{3}$ Institute of Evolution, University of Haifa, Mount

7 Carmel, Haifa 31905, Israel.

8 *To whom correspondence should be addressed. E-mail: lingonghua@ gmail.com

9 Abstract We report the twining handedness of Codonopsis pilosula, which has either a 10 left- or right-handed helix among different plants, among different tillers within a 11 single plant, and among different branches within a single tiller. The handedness was 12 randomly distributed among different plants, among the tillers within the same plants, 13 but not among the branches within the same tillers. Moreover, the handedness of the 14 stems can be strongly influenced by external forces, i.e. the compulsory left and right 15 forming inclined to produce more left- and right-handed twining stems, respectively, 16 and the reversing could make a left-handed stem to be right-handed and vice versa. We 17 also discuss the probable mechanisms these curious cases happen.

18 Keywords twining plant $\bullet$ left-handed $\bullet$ right-handed $\bullet$ external force

\section{Introduction}

The graceful movements of twining plants have fascinated biologists since Charles

21 Darwin's time (Darwin, 1865; Isnard and Silk, 2009). Most of the twining plants show

22 fixed handedness, either consistently forming right- or left-handed helices as they

23 climb, which depends on the genus and/or species (Hashimoto, 2002). Although very

24 rare, there are also some exceptions, e.g. in Dioscorea and Wisteria, where different 
species twine in one direction or the other (Ornduff 1991).

Darwin (1865) also provided cases of different individuals in the same species twined in both directions, and even, some of the individuals twined firstly in one direction and then reversed their course. However, as Darwin mentioned, the stems of these species could be easily pulled from its support with little unwinding. Hence, these species should be more suitable to be viewed as climbing than twining plants.

Codonopsis pilosula (Campanulaceae) is a typical twining plant native to China and surrounding areas and usually found growing around forest openings under the shade of trees. Both left- and right- handed stems of this species are found (Fig. 1 A, B). Also, different tillers within a single plant (Fig. $1 \mathrm{C}$ ) and different branches in a single tiller (Fig. 1 D, E) may twine in both directions. Moreover, once a stem (tiller or branch) starts the twining, it will not change direction unless with compulsory force (see below), also different from the so called ambidextrous twining (Burnham and Revilla-Minaya 2011). This makes it a good candidate to statistically analyze the handedness nature of twining plants. Here, based on hundreds of observations, we aimed to answer two rudimentary questions we are interested: 1) Are the handedness of the tillers of this species happened randomly? 2) Can external forces influence the handedness of the tillers?

\section{Materials and Methods}

\section{General observation}

The $C$. pilosula seedlings were planted and in the spring on the second year, before the plants were germinated, each plant was provided with a PVC pipe (1.8 meters long and the diameter is $16 \mathrm{~mm}$ ) as the supporting material.

Five hundred C. pilosula were planted and kept intact for the general observation of handedness distribution. The first growing tiller of each plant was used for the 
statistical analysis on handedness distribution among plants. Plants having two or more

51 tillers were observed for handedness distribution statistical analysis within each plant. The first growing tillers having six or more branches were used for the handedness distribution of their branches and their relationship with the mother tillers themselves.

\section{Compulsory treating}

Two hundred $C$. pilosula were planted, in which each half were compulsorily fixed as left- and right-handed, respectively, by adhesive cellophane on the tip of the youngest leaf to the supporting PVC pipes. The fixation was executed before the stems have started their own twining behaviors and continued following the growing of the stems until the plants covering two helix pitches, we then removed the adhesion material and kept the plants growing freely again. The handedness of each stem was recorded when it gained another two or more helix pitches.

Eighty more $C$. pilosula were planted for compulsorily reversing treat (one tiller for each plant). First, the tillers were kept intact to grow for more than two helix pitches and then we reversed their initial direction until the young stem showed fixation of their handedness (usually 1.5 3 helixes) using adhesive cellophane. We then removed the adhesion material and kept the plants growing freely again. The twining handedness of each stem was recorded when it gained another two or more helix pitches.

\section{Statistical analysis}

We used "Chi-square goodness of fit tests" in Minitab 16.0 to test whether the left vs. right ratio among freely grown tillers and compulsorily fixed tillers deviate from the 1:1 ratio. For the ratios among tillers within the plants and among branches within tillers, the nonparametric "2 Related Samples test" in SPSS 20.0 was used.

\section{Results}

A total of 459 plants (others were dead or stopped growing because of root rot or aphid 
feeding) were observed, and the handedness of the first twined tiller for each plant was recorded for handedness distribution analysis among plants. 216 tillers were left-handed and 243 were right-handed, not significantly deviate from the 1:1 ratio ( $x$ $\left.{ }^{2}=1.588, d f=1, P=0.208\right)$. There were 210 plants that had two or more tillers, and the ratio of left- and right-handed tillers within the same plants were $0.488 \pm 0.348$ and $0.512 \pm 0.348$, respectively, also not significantly deviate from the $1: 1$ ratio $(Z=-0.613$, $N=210, P=0.540)$.

Eighty tillers (forty for left- and forty for right-handed) which had six or more twined branches were selected to test whether the handedness of mother tillers can affect the handedness of their branches. For the left-handed tillers, the proportion of left- and right-handed branches were $0.685 \pm 0.211$ and $0.315 \pm 0.211$, respectively, significantly deviated from the $1: 1$ ratio $(Z=-4.308, N=40, P<0.001)$; while for the right-handed tillers, the proportion of left- and right-handed branches were $0.273 \pm$ 0.149 and $0.727 \pm 0.149$, respectively, also significantly deviated from the $1: 1$ ratio $(Z=$ $-5.188, N=40, P<0.001)$.

Ninety-two and eighty-one compulsory left- and right-handed forming tillers successfully grow for more than two helix pitches after treating, respectively. From the compulsorily left-handed-formed tillers, eighty-four continued to be left-handed while eight reversed to right-handed, significantly deviated from the $1: 1$ ratio $\left(x^{2}=62.783\right.$, $d f=1, P<0.001)$. From the compulsorily right-handed-formed tillers, seventy-four continued to be right-handed while seven reversed to right-handed, also significantly deviated from the 1:1 ratio $\left(x^{2}=55.420, d f=1, P<0.001\right)$.

Thirty and thirty-four tillers successfully grow for more than two helix pitches after left-to-right and right-to-left treating, respectively (Fig. $1 \mathrm{~F}, \mathrm{G})$. For the left-to-right 
reversing group, twenty-three became right-handed while seven kept to be left-handed, significantly deviated from the $1: 1$ ratio $\left(x^{2}=8.533, d f=1, P<0.01\right)$. For the right-to-left reversing group, twenty-nine became left-handed while five kept to be right-handed, also significantly deviated from the 1:1 ratio $\left(x^{2}=9.529, d f=1, P<0.01\right)$.

\section{Discussion}

C. pilosula is hitherto one of the most variable plants on twining handedness i.e. different handedness can be found among different plants, among the tillers with the same plants, and among the branches within the same tillers. Our results also showed that, the handedness was randomly distributed among plants and among tillers within plant individuals, while the handedness of branches are positively influenced by their mother tillers. Moreover, the compulsory forming and reversing experiments in our study provided curious cases, i.e. the handedness of the stems can be strongly influenced by external forces.

Thigmonastic reactions are thought to be responsible for winding behaviors of twining plant (Darwin \& Darwin, 1881; Burnham and Revilla-Minaya, 2011). We presume a possible mechanism: stems are moving in a circle in the air looking for a support, at the moment they touch an object, the point of touch influenced growth hormone distribution between the inner and outer parts and lead to asymmetric growth. In that way they create helices. For most twining plants, the stems can only move with single directions (sinistrally or dextrally) and finally form left- and right-handed helices, respectively. Interestingly however, for C. pilosula, based on our observations, the young stems (or tillers) can move either dextrally or sinistrally, that is why they have different handedness.

The positive effects of the handedness of mother tillers on their branches might work by two mechanisms. Take left-handed tiller and their branches as example, the first 
mechanism might be: the branches from left-handed tillers incline to make sinistral circles and touch the supporters by their right sides and finally form left-handed helices. The second mechanism might be: the branches from left-handed tillers have equal

127 probability of moving directions but incline to lodge to the left and then have more 128 chances to touch the supporters by their right sides. Although more experimental 129 observations are needed to test our hypotheses, we suggest the sides where the stems 130 (both tillers and branches) meet and touch the supporters are critical to determine their 131 final handedness. The effects of external forces can also be explained by the 132 thigmotropism hypothesis, e.g. the compulsorily left-handed forming and left-to-right 133 reversing enhanced the touch between supporters and the right / left side of stems and 134 as a result, forming / reversing their final handedness.

135 With the development of biotechnology, studies on winding related mechanisms are 136 also moving to molecular cell levels. Hatakeda et al (2003) reported a Japanese strain of 137 morning glory (Pharbitis nil) whose shoots display weeping growth. Further studies 138 showed that, gravisensing endodermal cells are indispensable for the winding response 139 and that morning glory SCARECROW gene is responsible for the abnormal 140 phenotypes of weeping (Kitazawa et al 2005). However, because the morning glory is a 141 right-handed plant, these studies can only tell why the stem wind or not. Another study 142 on mutant Arabidopsis thaliana (including left- and right-handed mutants) indicated 143 that microtubule cytoskeleton might be critical for determining cell shape and thus the 144 stem handedness (Hashimoto, 2011). Because the $A$. thaliana is not a twining plant, we 145 suggest studying on the C. pilosula will provide more direct and convincing evidences.

146 It should be mentioned that, $C$. pilosula is also an important traditional Chinese 147 medicine. The cultivation related experiences and studies will also facilitate further 148 analysis in the lab. 
149 Acknowledgments We thank Takashi Hashimoto, Will Edwards, Avigdor Beiles, and

150 Yang Zhong for their valuable comments. We also thank Robin Permut for her

151 assistance in editing. This work was supported by the CAS Special Grant for

152 Postgraduate Research, Innovation and Practice (To G. Lin).

153 References

154 Burnham RJ, Revilla-Minaya C (2011) Phylogenetic influence on twining chirality in 155 lianas from Amazonian Peru. Ann Mo Bot Gard 98(2):196-205

156 Darwin C, Darwin F (1880) The power of movement in plants. John Murray, London

157 Darwin C (1865) On the movements and habits of climbing plants. Bot J Linn Soc $1589: 1-118$.

159 Hashimoto T (2011) Microtubule and cell shape determination. In: Liu B. The plant $160 \quad$ cytoskeleton. Springer, New York, pp 245-257

161 Hashimoto T (2002) Molecular genetic analysis of left-right handedness in plants. Phil

162 Trans Roy Soc B- Biol Sci 357 (1422):799-808

163 Hatakeda Y, Kamada M, Goto N, Fukaki H, Tasaka M, Suge H, Takahashi H (2003)

164 Gravitropic response plays an important role in the nutational movements of the 165 shoots of Pharbitis nil and Arabidopsis thaliana. Physiol Plant 118:464-473

166 Isnard S, Silk WK (2009) Moving with climbing plants from Charles Darwin's time 167 into the 21st century. Amer J Bot 96(7):1205-1221

168 Kitazawa D, Hatakeda Y, Kamada M, Fujii N, Miyazawa Y, Hoshino A, Iida S, Fukaki

169 H, Morita MT, Tasaka M, Suge H, Takahashi H (2005) Shoot circumnutation and 170 winding movements require gravisensing cells. Proc Nat Acad Sci USA $171 \quad 102(51): 18742-18747$

172 Ornduff R (1991) Handedness in twining tracheophytes. Amer J Bot 78:208 173 


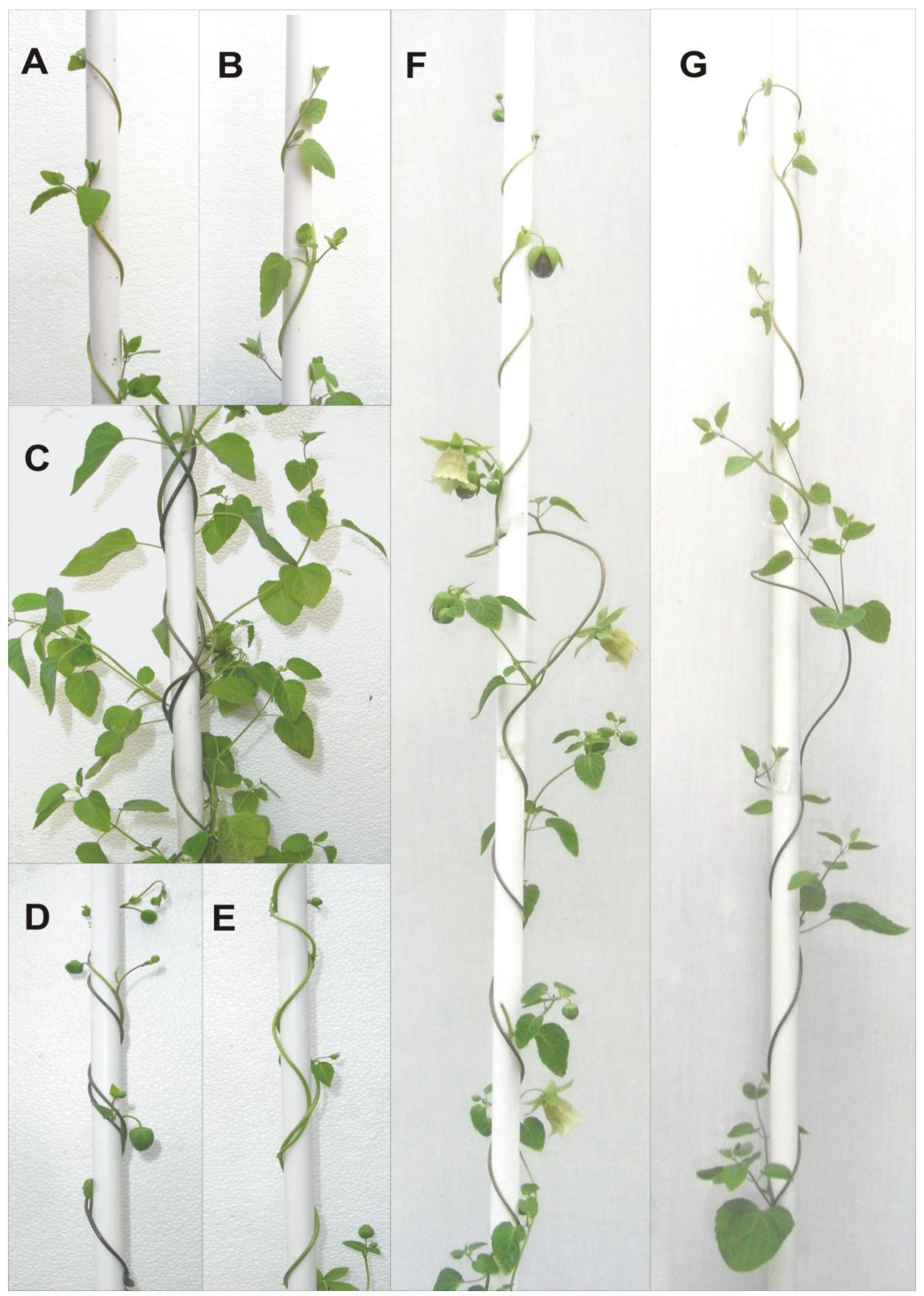

175 Fig. 1. Handedness of Codonopsis pilosula. (A) Left-handed. (B) Right-handed. (C) Both left- and 176 right-handedness in a single plant. (D) A left-handed (mother) tiller with a left-handed branch. (E) A right-handed tiller with a left-handed branch. (F) Revered from left-handed to right-handed. (G) Revered

178 from right-handed to left-handed. 